\title{
AVALIAÇÃO DOS CUSTOS DE ENERGIA ELÉTRICA PARA A INDÚSTRIA*
}

\author{
José Ronaldo Silveira Júnior ${ }^{1}$ \\ Henrique Ferreira Morici \\ Danilo Iglesias Brandão ${ }^{3}$ \\ Sidelmo Magalhães Silva ${ }^{4}$
}

\section{Resumo}

O objetivo deste trabalho é de identificar estratégias para avaliação da conta de energia de unidades industriais, que sirvam de subsídio para o processo de tomadas de decisão e que eventualmente forneçam benefícios econômicos imediatos, sem investimento. Para esse fim, aplica-se uma metodologia simples que consiste em três aspectos: avaliação da demanda contratada, análise de modicidade tarifária e averiguação de excesso de consumo de energia reativa. Apresenta-se um estudo de caso da aplicação dessa metodologia em uma unidade produtiva de distribuição de aço. Nesse estudo, observa-se redução de custos por se evitar penalidades tanto de ultrapassagem de demanda quanto de excesso de energia reativa, representando uma economia de aproximadamente $15 \%$ do valor total da conta de energia elétrica da unidade.

Palavras-chave: Análise Tarifária de Energia; Demanda Contratada; Eficiência Energética; Fator de Potência; Potência Reativa.

\begin{abstract}
EVALUATING OF THE ELECTRICITY COSTS FOR THE INDUSTRY Abstract

The goal of this paper is to identify strategies for evaluating the electricity bill of industrial plants, to subsidize the decision making process and eventually provide economic benefits without any investment. Thus, a simple methodology is devised and it consists of three aspects: contracted demand evaluation, tariff modality analysis and reactive energy overconsumption scrutiny. A case study is presented, comprising the applied methodology to a productive unity. In this study, the cost reduction is verified due to avoiding penalties for exceeding both demand and reactive energy limits, which represents approximately $15 \%$ of the total electricity bill for this industrial plant.
\end{abstract}

Keywords: Contracted Demand; Energy Efficiency; Power Factor; Reactive Power; Tariff Analysis.

1 Engenheiro Eletricista, Mestre, Estudante, Universidade Federal de Minas Gerais, Belo Horizonte, MG, Brasil.

2 Engenheiro Eletricista, Estudante, Universidade Federal de Minas Gerais, Belo Horizonte, MG, Brasil.

3 Engenheiro de Controle e Automação, Doutor, Professor, Universidade Federal de Minas Gerais, Belo Horizonte, MG, Brasil

4 Engenheiro Eletricista, Doutor, Professor, Universidade Federal de Minas Gerais, Belo Horizonte, MG, Brasil. 


\section{INTRODUÇÃO}

O custo de energia elétrica está entre os mais significativos durante as etapas de transformação e agregação de valor da indústria brasileira, em especial a cadeia metalúrgica. Dessa forma é fundamental adotar medidas que visem eliminar desperdícios, reduzir custos e aumentar a eficiência do consumo de energia, de modo a tornar a indústria mais competitiva e ao mesmo tempo, mais sustentável.

Estudos de avaliação do consumo e demanda, análise de modalidade tarifária e correção de fator de potência são algumas das maneiras eficientes de se reduzirem os custos com energia. Muitas vezes são avaliações realizadas por empresas de consultoria, mas que tem condições de serem realizadas pela própria empresa de maneira mais confiável e econômica. Nesse sentido esse trabalho propõe um guia prático e rápido para avaliação da conta de energia de forma independente e confiável, à luz da legislação corrente do setor elétrico. Serão avaliados três itens básicos da conta de energia de uma indústria, a saber: a demanda contratada para redefinir o valor adequado a fim de eliminar a cobrança de penalidades ou evitar sobrecontratação, efetuar uma análise tarifária para estabelecer a modicidade adequada para a unidade no ambiente de contratação cativo e averiguar o excesso de energia reativa para dimensionar banco de capacitores para corrigir o fator de potência.

\subsection{Contextualização e Conceitos Básicos}

A fim de compreender os problemas e objetivos propostos, serão apresentados alguns conceitos básicos frequentemente tratados na regulamentação de energia e nos procedimentos de distribuição. Esses permitirão uma compreensão básica da metodologia aplicada nesse trabalho.

\subsubsection{Demanda e Energia}

O valor da conta de energia depende basicamente de dois elementos: demanda e energia [1]. São elementos distintos, representados inclusive por unidades de medida distintas, mas que frequentemente são confundidos.

O termo "Demanda", corresponde à potência média verificada a cada intervalo de 15 minutos. A potência é a capacidade de consumo de uma planta ou de um equipamento, representado, por exemplo, pelo valor que vem especificado, na compra de aparelhos eletrônicos. É expressa em watts (W) e seus múltiplos.

A demanda contratada é o valor de demanda (potência) máxima fixada em contrato no qual a concessionária se compromete garantir ao consumidor uma disponibilidade de rede. Essa disponibilidade exige um custo que faz parte da fórmula de tarifação da conta, correspondendo a uma parcela fixa. Esse custo fixo representa um "aluguel de espaço" na rede elétrica para o consumidor.

$O$ adequado é que a demanda apurada seja menor ou igual àquela contratada (quanto mais próxima melhor), sendo faturado o maior valor entre o contratado e 0 efetivamente registrado. Caso o consumidor exceda o valor contratado, salvo uma tolerância de $5 \%$, a ultrapassagem é tarifada com valor três vezes maior, conforme legislação em vigor [3-4].

Já a energia pode ser entendida como a quantidade de eletricidade utilizada por um equipamento em um determinado tempo. A unidade é o watt-hora (Wh) ou seus múltiplos, representando basicamente a potência multiplicada pelo número de 
horas de utilização. O custo com a energia não apresenta valor fixo e dependerá do consumo apurado no mês, constituindo uma parcela variável no valor da conta total.

\subsubsection{Horários de Ponta e Fora Ponta}

Existe uma diferenciação entre as horas do dia para efeitos de tarifação. $O$ horário de ponta (HP) é constituído por três horas consecutivas, exceto fins de semana e feriado, nos quais o sistema elétrico é mais requisitado. O horário fora de ponta (HFP) corresponde às demais horas do dia. O HP é definido por cada concessionária conforme características particulares. Em Minas Gerais, por exemplo, é definido para a Cemig o HP das 17 às 20 horas. Com horário de verão as horas de ponta são de 18 às 21 horas. Geralmente, os preços de demanda e/ou energia possuem valor mais elevado no HP. Esse ponto é um fator determinante para determinar a modalidade ideal, conforme o padrão de consumo de cada unidade.

\subsubsection{Potência Ativa e Reativa}

A potência gerada nas usinas é chamada de potência aparente $(S)$, porém essa se divide nas chamadas potência ativa $(P)$ e reativa (Q). A potência ativa é responsável pela realização de trabalho útil, isso significa movimentar um motor, acionar uma máquina, gerar calor, prover iluminação, etc. Já a potência reativa não produz trabalho, somente circula entre a carga consumidora e a fonte elétrica. A potência reativa circulante aumenta o valor eficaz da corrente total, podendo sobrecarregar as linhas de distribuição e transmissão, e aumentar as perdas Joules [2]. A redução da potência reativa circulante está relacionada à eficientização energética e muito embora tenha grande utilidade prática, relacionada a magnetização, a potência reativa é uma grandeza não física.

A potência aparente é a soma quadrática entre $P$ e $Q$, pois essas quantidades são ortogonais, conforme Equação 1:

$$
\mathrm{S}^{2}=\mathrm{P}^{2}+\mathrm{Q}^{2}
$$

Devido à relação entre essas grandezas dadas pela Equação 1, torna-se mais compreensível lidar com as mesmas sob a forma de um triângulo retângulo, usualmente conhecido como "triângulo das potências", conforme a Figura 1.

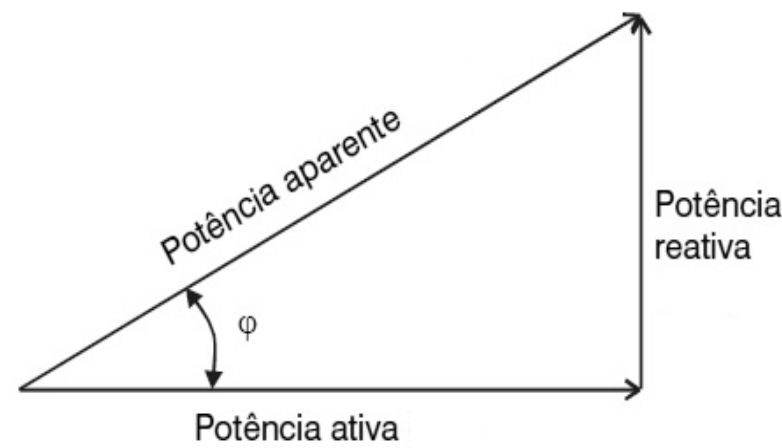

Figura 1. Triângulo de potências. 
Necessita-se que a potência reativa seja mínima, representando um cateto oposto a $\varphi$ com a menor altura possível. Idealmente não há nenhum consumo de reativo e a potência ativa se iguala à aparente. Uma forma de avaliar essa "eficiência" é calcular o cosseno de $\varphi$, chamado de fator de deslocamento ou fator de potência (FP) (Equação 2):

$$
F P=\cos \varphi=\frac{P}{S}
$$

Quanto mais próximo for o FP da unidade, melhor a qualidade de energia elétrica. A Agência Nacional de Energia Elétrica (ANEEL) estabelece em [3]-[4] o valor mínimo de FP de 0,92 para consumidores industriais a níveis de tensão de até 13,8KV. Caso esse fator esteja abaixo de 0,92, ocorre cobrança de penalidade reativa.

Uma forma de entendimento é que circulação de reativo elevado reduzirá a quantidade de potência ativa consumida para uma mesma potência aparente, dessa forma o termo "pagamento de reativo" amplamente visto na indústria não é utilizado corretamente, haja vista que paga-se na verdade pela potência ativa que a concessionária deixou de receber em função do FP abaixo do permitido pela legislação.

\section{MATERIAIS E MÉTODOS}

A metodologia proposta será composta de três etapas de avaliação da conta de energia de unidades industriais, à luz da legislação do setor elétrico, a saber:

\section{- Demanda contratada:}

Consistirá na avaliação da demanda contratada para redefinir o valor adequado a fim de eliminar a cobrança de penalidades ou evitar sobrecontratação. Para a redefinição é necessário um alinhamento entre a análise histórica dos valores registrados de demanda e a perspectiva futura de atividade industrial;

- Análise de Modicidade Tarifária:

Consistirá de uma análise das modalidades tarifárias existentes no ambiente cativo para sistemas de distribuição, a fim estabelecer a modicidade adequada para a unidade. A análise tarifária requer a identificação de quatro grandezas: demanda (definida no item anterior) e energia nos horários de ponta e fora ponta. Serão consideradas as tarifas THS (Tarifa Horo Sazonal) Verde e THS Azul [1]. Na modalidade Verde, há tarifa de demanda única e tarifas de energia distintas para HP e HFP. Já a Azul determina tarifas distintas tanto para demanda quanto energia em HP e HFP. As tarifas das modalidades Verde e Azul são reguladas por resolução [5]. Matematicamente, os consumos de demanda $D$ e de energia $E$, multiplicados pelas tarifas de demanda TD e de energia TE, determinam os valores a pagar em cada modalidade, conforme a Equação 3 e 4:

$$
\begin{aligned}
& \text { Valor }(\text { Verde })=D_{\text {única }} \cdot T D_{\text {única }} \text { verde }+E_{H F P} \cdot T E_{H F P_{\text {verde }}}+E_{H P} \cdot T E_{H P_{\text {verde }}} \\
& \operatorname{Valor}(A z u l)=D_{H F P} \cdot T D_{H F P_{a z u l}}+D_{H P} \cdot T D_{H P_{a z u l}}+E_{H F P} \cdot T E_{H F P_{a z u l}}+E_{H P} \cdot T E_{H P_{a z u l}}
\end{aligned}
$$


- Reativo:

Consistirá em averiguar o excesso de potência reativa para dimensionar o banco de capacitores para correção do fator de potência, caso seja necessário. Conforme [3], a multa por reativo $\mathrm{E}_{\mathrm{RE}}$ em reais (Equação 5) depende do montante de energia elétrica ativa EEAM medida no faturamento, do fator de potência médio $f_{M}$ medido na unidade e da tarifa de energia na bandeira verde VRER.

$$
E_{R E}=E E A M \cdot\left(\frac{0,92}{f_{M}}-1\right) \cdot V R_{E R E}
$$

Essa formulação basicamente precifica a diferença de energia ativa que deveria ter sido consumido pela unidade a um FP de 0,92 para a mesma energia aparente fornecida, mas que na realidade se tornou excesso de energia reativa devido ao baixo valor de FP. A necessidade de consumo de potência ativa seria a mesma com um FP adequado, mas a potência aparente entregue seria menor. Ou seja, paga-se por consumo ativo maior do que realmente necessário, para compensar o fato de a rede elétrica transportar reativo desnecessário. De forma prática, a Equação 6 permite obter o FP registrado isolando-se o termo $f_{M}$ :

$$
\mathrm{f}_{\mathrm{M}}=\left(0,92 \cdot E E A M \cdot V R_{E R E}\right) /\left(E_{R E}+E E A M \cdot V R_{E R E}\right)
$$

De forma geral, as causas principais para um baixo fator de potência são: utilização de motores/transformadores a vazio ou a pequenas cargas, grande quantidade de máquinas com pequena potência, utilização de máquinas de solda ou não utilização de reator em iluminação com lâmpadas fluorescentes.

O banco de capacitores que corrige o problema compensa o excesso de potência reativa circulante. Geometricamente, o triângulo das potências atual precisa ser alterado para um novo, com mesma base e menor altura. Isso mantém a potência ativa atual requerida pela planta, mas reduz a potência reativa, adequando o FP para 0,92. A Figura 2 ilustra essa alteração.
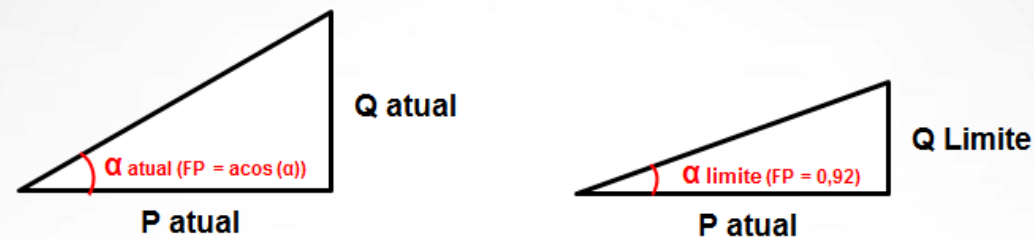

Figura 2. Alteração do triângulo de potências para correção de FP

O equipamento deve compensar a diferença entre as alturas dos dois triângulos da Figura 2. A partir de cálculos geométricos e trigonométricos simples, 0 dimensionamento do banco de capacitores $C$ pode ser feito pela Equação 7, tendo como unidade de grandeza o volt-ampère reativo (VAr) e seus múltiplos.

$$
C=P_{\text {atual }} \cdot[\tan (\operatorname{acos}(f p))-\tan (\operatorname{acos}(0,92))]
$$

$P_{\text {atual }}$ pode ser considerado como a potência média consumida, isto é, a energia faturada dividida pelo número de horas do mês. A partir da Equação 7, são calculados os bancos para corrigir os FP Após os cálculos valores comerciais dos equipamentos devem ser adquiridos e instalados para sanar o pagamento devido ao baixo valor de fator de potência. 


\section{RESULTADOS E DISCUSSÃO}

Como cenário para aplicação da metodologia proposta foram utilizadas as informações físicas e financeiras reais relacionadas à energia de uma unidade industrial de distribuição de aço, localizada no estado de Minas Gerais. Essa unidade possui demanda contratada de $123 \mathrm{~kW}$ e está na modalidade tarifária verde e no ambiente cativo de contratação de energia. A conta de energia mensal fica em torno de $R \$ 50.000$ por mês, incluindo impostos.

\subsection{Adequação de Demanda Contratada}

A Figura 3 ilustra as demandas contratada e registrada em $\mathrm{kW}$ para ambos os horários HFP e HP. Observa-se que em todos os meses analisados há ultrapassagem do valor contratado. Para $123 \mathrm{~kW}$ contratados, as médias registradas são de 226 kW no HFP e de 221 kW HP.

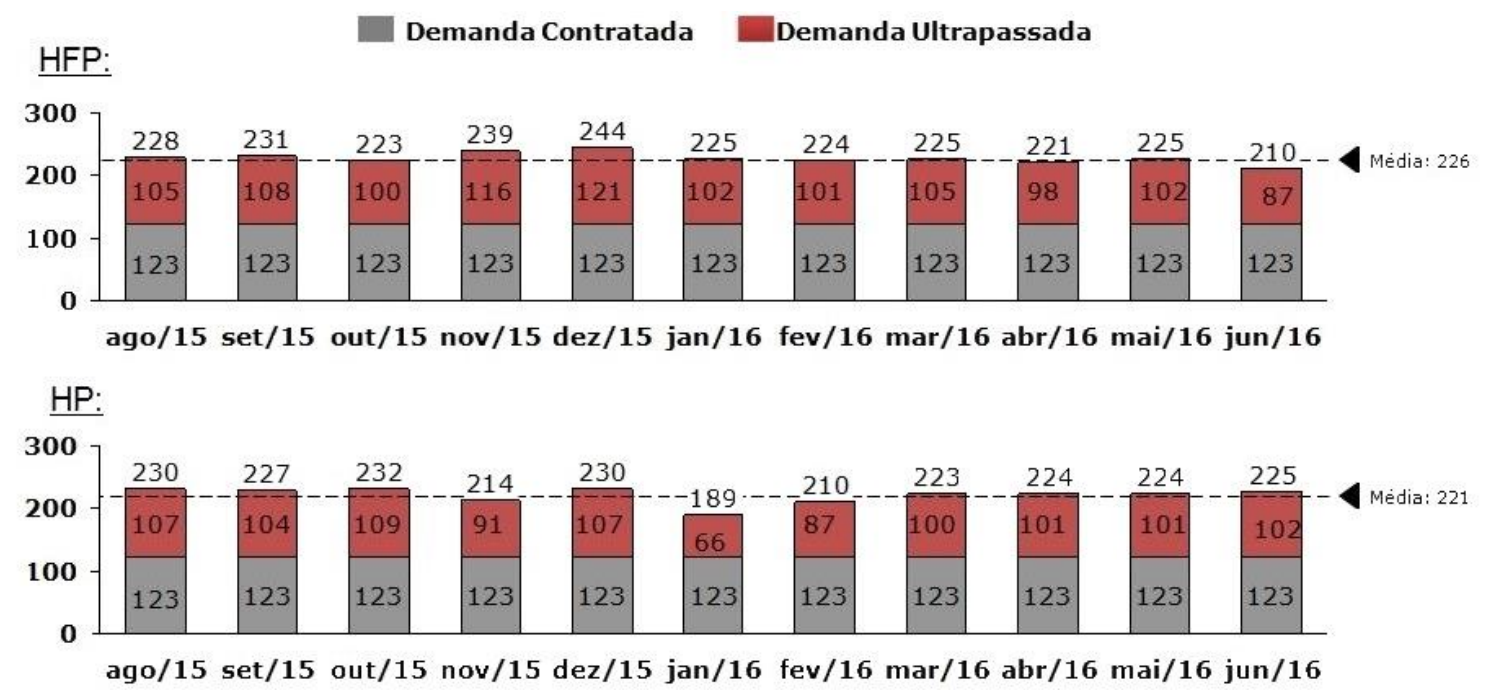

Figura 3. Demandas contratada e ultrapassada.

Os valores em vermelho sinalizam a ultrapassagem de demanda, havendo incidência de uma tarifa três vezes mais cara para essa parcela. Para a redefinição, foi analisado o histórico de consumo e alinhamento com a unidade fabril sobre a perspectiva futura de atividade industrial. Neste caso, concluiu-se que uma demanda contratada de 260 kW para HFP e HP satisfaria as necessidades da planta e eliminaria o problema das ultrapassagens. Nesses casos pode ser avaliado o uso dos $5 \%$ adicionais de demanda permitidos para uso além do contratado sem que não haja multa por ultrapassagem. A solicitação de aumento foi feita à concessionária que atendeu de imediato, uma vez que não houve necessidades de obras. Para reduções há uma carência de 180 dias para que efetivamente se perceba o valor menor. Em ambos os casos o coordenograma de proteção precisa ser ajustado à nova realidade. A redefinição foi autorizada pela distribuidora de energia e a partir do mês subsequente observou o benefício, conforme a Tabela 1. 
Tabela 1. Pagamento de penalidades por ultrapassagem de demanda

\begin{tabular}{|c|c|c|c|}
\hline Meses & Penalidade & Mês & Penalidade \\
\hline ago/15 & $\mathrm{R} \$ 2.554$ & $\mathrm{mar} / 16$ & $\mathrm{R} \$ 2.396$ \\
\hline set/15 & $\mathrm{R} \$ 2.605$ & $a b r / 16$ & $R \$ 2.309$ \\
\hline out $/ 15$ & $\mathrm{R} \$ 2.626$ & mai/16 & $R \$ 2.402$ \\
\hline nov/15 & $\mathrm{R} \$ 2.755$ & jun/16 & $\mathrm{R} \$ 2.409$ \\
\hline $\mathrm{dez} / 15$ & $\mathrm{R} \$ 2.836$ & jul/16 & $\mathrm{R} \$ 0$ \\
\hline $\mathrm{jan} / 16$ & $R \$ 2.400$ & ago/16 & $\mathrm{R} \$ 0$ \\
\hline $\mathrm{fev} / 16$ & $\mathrm{R} \$ 2.433$ & set/16 & $\mathrm{R} \$ 0$ \\
\hline
\end{tabular}

Para avaliação do benefício médio obtido deve-se considerar a penalidade menos o aumento de custo com a adequação da contratação. Assim, compara-se os custos totais relativos ao antigo valor de demanda (penalidade inclusa), e os custos totais relativos ao novo valor (sem penalidades). Para meses anteriores à alteração, deve-se calcular qual seria o custo caso a demanda fosse o novo valor, sem penalidades. Para meses posteriores à alteração, deve-se calcular qual seria o custo caso a demanda fosse o antigo valor, com penalidades. Esses cálculos são representados na (Tabela 2), que registra o benefício médio mensal apurado de $R \$$ 2.116 ou $\mathbf{R} \$ \mathbf{2 5 . 3 9 2}$ anual.

Tabela 2. Apuração do benefício mensal com a readequação de demanda

\begin{tabular}{cccc}
\hline Mês & $\begin{array}{c}\text { Custo Demanda } \\
\text { Antiga }\end{array}$ & $\begin{array}{c}\text { Custo Nova } \\
\text { Demanda }\end{array}$ & Benefício \\
\hline ago/15 & $\mathrm{R} \$ 5.298$ & $\mathrm{R} \$ 3.103$ & $\mathrm{R} \$ 2.196$ \\
\hline $\mathrm{set} / 15$ & $\mathrm{R} \$ 5.386$ & $\mathrm{R} \$ 3.133$ & $\mathrm{R} \$ 2.253$ \\
\hline $\mathrm{out} / 15$ & $\mathrm{R} \$ 5.420$ & $\mathrm{R} \$ 3.132$ & $\mathrm{R} \$ 2.289$ \\
\hline $\mathrm{nov} / 15$ & $\mathrm{R} \$ 5.592$ & $\mathrm{R} \$ 3.087$ & $\mathrm{R} \$ 2.505$ \\
\hline $\mathrm{dez} / 15$ & $\mathrm{R} \$ 5.696$ & $\mathrm{R} \$ 3.047$ & $\mathrm{R} \$ 2.649$ \\
\hline $\mathrm{jan} / 16$ & $\mathrm{R} \$ 5.047$ & $\mathrm{R} \$ 3.059$ & $\mathrm{R} \$ 1.988$ \\
\hline $\mathrm{fev} / 16$ & $\mathrm{R} \$ 5.131$ & $\mathrm{R} \$ 3.132$ & $\mathrm{R} \$ 1.999$ \\
\hline $\mathrm{mar} / 16$ & $\mathrm{R} \$ 5.039$ & $\mathrm{R} \$ 3.054$ & $\mathrm{R} \$ 1.985$ \\
\hline $\mathrm{abr} / 16$ & $\mathrm{R} \$ 4.883$ & $\mathrm{R} \$ 2.987$ & $\mathrm{R} \$ 1.895$ \\
\hline $\mathrm{mai} / 16$ & $\mathrm{R} \$ 5.065$ & $\mathrm{R} \$ 3.078$ & $\mathrm{R} \$ 1.988$ \\
\hline $\mathrm{jun} / 16$ & $\mathrm{R} \$ 5.081$ & $\mathrm{R} \$ 3.087$ & $\mathrm{R} \$ 1.994$ \\
\hline $\mathrm{jul} / 16$ & $\mathrm{R} \$ 4.299$ & $\mathrm{R} \$ 2.569$ & $\mathrm{R} \$ 1.729$ \\
\hline $\mathrm{ago} / 16$ & $\mathrm{R} \$ 4.494$ & $\mathrm{R} \$ 2.375$ & $\mathrm{R} \$ 2.119$ \\
\hline set/16 & $\mathrm{R} \$ 4.487$ & $\mathrm{R} \$ 2.371$ & $\mathrm{R} \$ 2.116$ \\
\hline MÉDIA & $\mathrm{R} \$ 5.066$ & $\mathbf{R} \$ 2.944$ & $\mathbf{R} \$ 2.122$ \\
\hline
\end{tabular}

\subsection{Análise de Modicidade Tarifária}

Para a análise de modicidade tarifária, usou-se o valor redefinido de demanda do item 3.1 e a média dos consumos de energia presentes nas faturas, que se encontram na Tabela 3.

Tabela 3. Demanda e Energia para a Análise Tarifária

\begin{tabular}{cccc}
\hline \multicolumn{2}{c}{ Demanda Contratada } & \multicolumn{2}{c}{ Consumo médio de Energia } \\
\hline $\mathrm{HFP}(\mathrm{kW})$ & $\mathrm{HP}(\mathrm{kW})$ & $\mathrm{HFP}(\mathrm{kWh})$ & $\mathrm{HP}(\mathrm{kWh})$ \\
\hline 260 & 260 & 64.400 & 9.262 \\
\hline
\end{tabular}


As tarifas das modalidades Verde e Azul são indicadas na Tabela 4 conforme publicação da Aneel (sem impostos). Assim, utilizando os dados da Tabela 3 e as Equações (3) e (4), encontra-se também na Tabela 4 os custos totais em cada modalidade.

Tabela 4. Tarifas reguladas Verde e Azul e custo total sem impostos

\begin{tabular}{|c|c|c|c|c|c|}
\hline & \multicolumn{2}{|c|}{ Demanda $(R \$ / k W)$} & \multicolumn{2}{|c|}{ Energia (R\$/kWh) } & \multirow{2}{*}{ Custo (R\$) } \\
\hline & HFP & $\mathrm{HP}$ & HFP & $\mathrm{HP}$ & \\
\hline THS Azul & 9 & 30 & 0,36476 & 0,50288 & $R \$ 38.288$ \\
\hline THS Verde & & & 0,36476 & 1,23 & $\mathrm{R} \$ 37.222$ \\
\hline
\end{tabular}

Observa-se que a tarifa Verde, que é a vigente, é a mais adequada nesse padrão de consumo. Porém, mais do que somente identificar a modalidade ideal para o consumo atual, é importante fazer uma análise de sensibilidade para comparar também o comportamento dos custos em cada tarifa com a variação desse consumo. Isso fornece subsídios mais sólidos para tomada de decisão da manutenção ou alteração da modalidade. Para efetuá-la, é feita uma simplificação: a demanda é fixada. Dessa forma, a análise de sensibilidade passa a depender somente dos valores de energia, reduzindo o problema de 4 para 2 variáveis.

Busca-se tornar essa dependência com uma única variável, para tornar a análise possível de ser identificada em um gráfico bidimensional. Assim, representase o consumo de um dos valores de energia (HP ou HFP) como uma proporção fixa do outro valor e o problema passa a ter somente 1 variável. Diante disso, são gerados 4 gráficos com situações distintas, a saber:

- Figura 4 representam-se os custos em cada tarifa em função do consumo no HFP. Promove-se uma variação tanto no consumo HFP quanto HP, mantendo a proporção fixa de HP para HFP igual à relação atual da Tabela 3 (aprox. $14,38 \%)$;

- A Figura 5 é semelhante, porém a proporção fixa entre energia HP para HFP adotada é o dobro da relação atual (28,76\%);

- Na Figura 6, apresentam-se as variações dos custos com a variação do consumo no HFP, mantendo fixo o consumo atual de HP;

- O caso da Figura 7 é o contrário da Figura 6: comparam-se os custos em função do consumo no HP, mantendo fixo o consumo atual de HFP. 


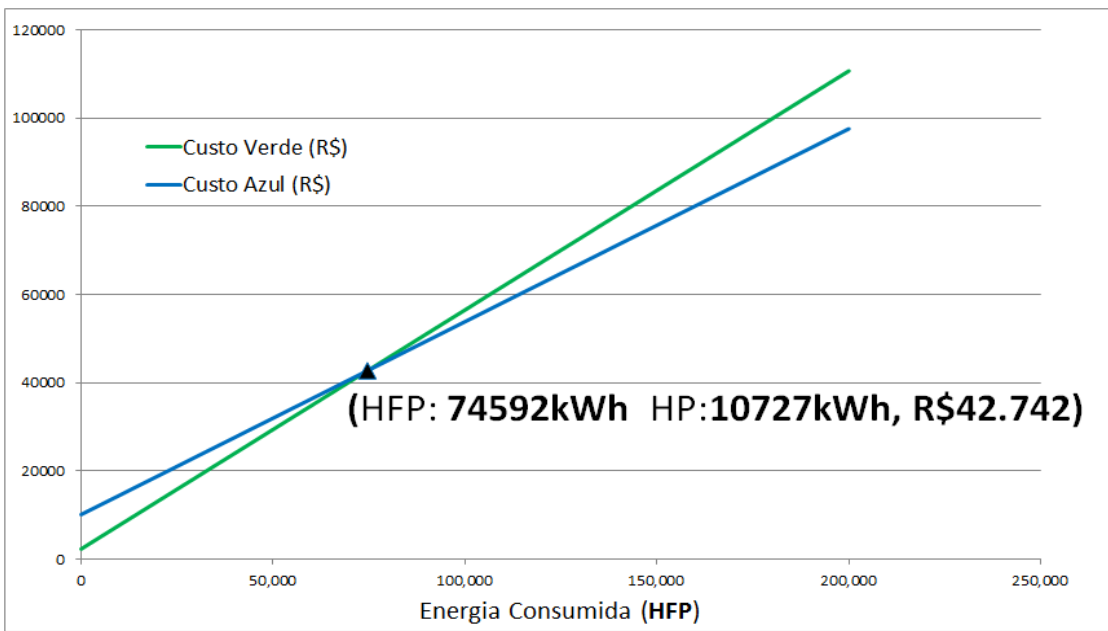

Figura 4. Custos x Energia HFP variando os consumos. Proporção: 14,38\% de HP

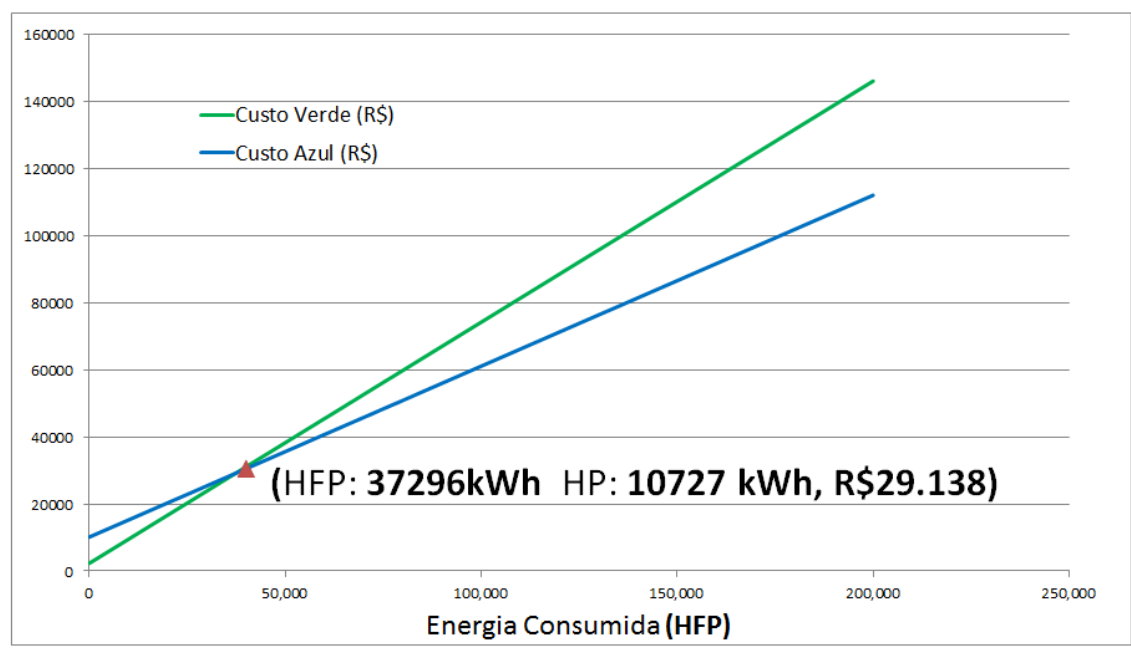

Figura 5. Custos x Energia HFP variando ambos os consumos. Proporção: $28,76 \%$ de HP

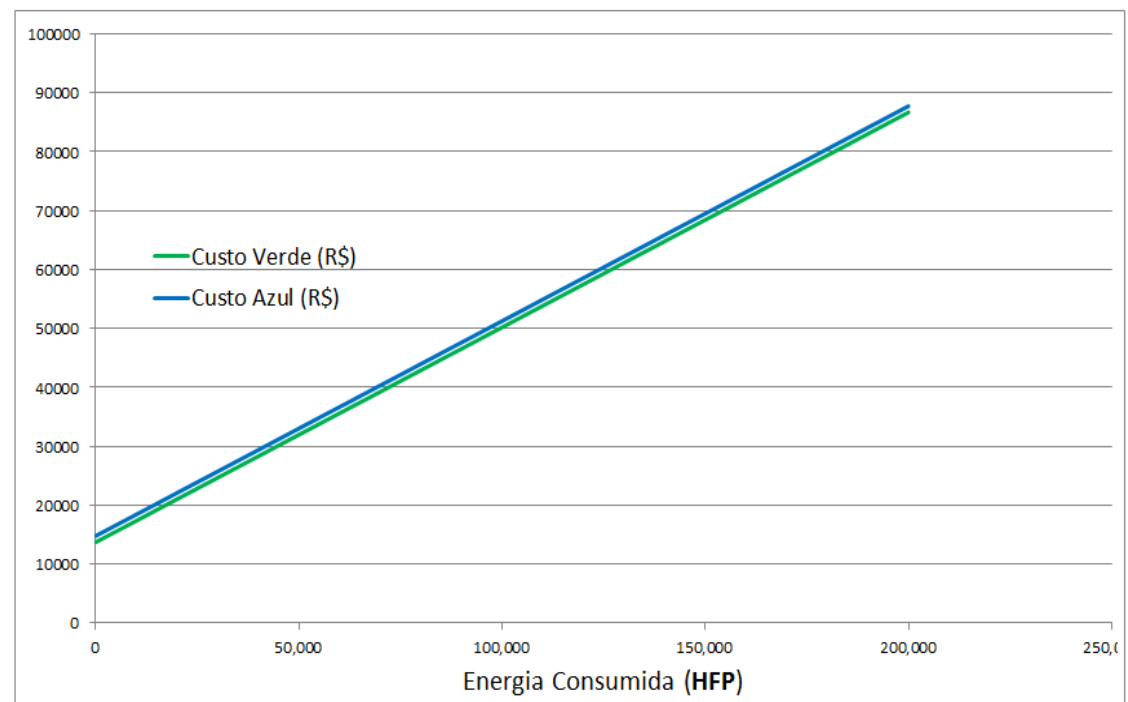

Figura 6. Custos $x$ Energia HFP variando o consumo HFP e mantendo fixo HP 


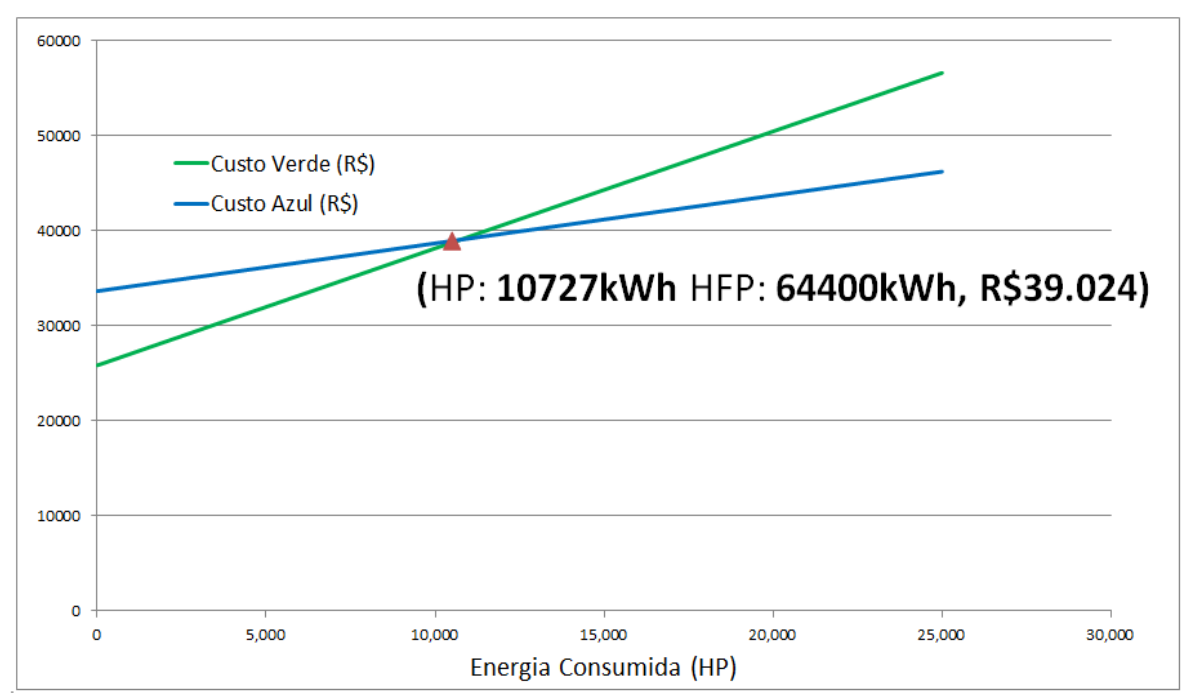

Figura 7. Custos x Energia HFP variando o consumo HP e mantendo fixo HFP

Todos os gráficos mostram que a modalidade Verde inicialmente é mais vantajosa. Porém, destaca-se que tanto na Figura 4 quanto na Figura 5, o ponto limite acima do qual a modalidade Azul tem melhor custo ocorre para o mesmo valor de consumo HP: $10.727 \mathrm{kWh}$. Ou seja, muito embora em cada situação o consumo HFP dessas condições seja diferente (devido às proporções entre HP e HFP diferentes), esses resultados demonstram que o consumo HP é determinante para a mudança de comportamento das modalidades, para as premissas adotadas. Isso é comprovado pela Figura 6, na qual se mantém o consumo atual HP e altera-se somente o consumo HFP. O custo Azul é sempre superior ao Verde (retas paralelas). Já quando há alteração somente do consumo HP, na Figura 7, existe ponto de interseção das curvas e esse se dá para a mesma condição de HP das Figuras 4 e 5: $10.727 \mathrm{kWh}$.

De posse dessa análise gráfica, aliados ao consumo médio HP da Tabela 3 (9.262 kWh) e dos custos atuais da Tabela 4, recomenda-se a manutenção da tarifa Verde. Caso esse valor ultrapasse o limiar de $10.727 \mathrm{kWh}$ (aumento superior a $15,8 \%$ ), recomenda-se a reavaliação da alteração para a modalidade Azul.

Uma outra abordagem é comparar as Equações (3) e (4). Para essa análise de sensibilidade, determina-se a condição dos consumos que torna o custo com a modalidade Azul menor do que a Verde. Toma-se a inequação (4) < (3) e, observando pela (Tabela 3) a existência de algumas tarifas de mesmo valor, obtémse a condição da (Equação 8):

$$
E_{H P}>\frac{D_{H P} \cdot T D_{H P_{a z u l}}}{T E_{H P_{\text {verde }}}-T E_{H P_{a z u l}}}
$$

A inequação 8 reforça que a decisão sobre a modalidade deve-se basear principalmente no consumo do HP. Existe um consumo limite que é função da demanda contratada no HP. Para valores abaixo desse limite deve-se optar pela modalidade Verde, e para valores acima, a Azul. Substituindo na Equação (8) a demanda de $260 \mathrm{~kW}$ e as tarifas de energia da Tabela 4, obtém-se o limite de 10.727 kWh no HP. 


\subsection{Correção de Fator de Potência}

Conforme mencionado fatores de potência abaixo de 0,92 são passíveis de penalidade. A Tabela 5 registra o histórico do pagamento das penalidades com reativo, onde se observa um pagamento médio de $R \$ 3.751$.

Tabela 5. Pagamento de penalidades por energia reativa

\begin{tabular}{|c|c|c|c|}
\hline Mês & Penalidade & Mês & Penalidade \\
\hline Mês 1 & $\mathrm{R} \$ 2.842$ & Mês 9 & $\mathrm{R} \$ 3.994$ \\
\hline Mês 2 & $R \$ 2.152$ & Mês 10 & $\mathrm{R} \$ 4.380$ \\
\hline Mês 3 & $\mathrm{R} \$ 2.241$ & Mês 11 & $R \$ 4.608$ \\
\hline Mês 4 & $\mathrm{R} \$ 2.253$ & Mês 12 & $\mathrm{R} \$ 4.603$ \\
\hline Mês 5 & $R \$ 1.962$ & Mês 13 & $\mathrm{R} \$ 5.286$ \\
\hline Mês 6 & $\mathrm{R} \$ 2.447$ & Mês 14 & $R \$ 5.033$ \\
\hline Mês 7 & $\mathrm{R} \$ 5.378$ & MÉDIA & $\mathrm{R} \$ 3.751$ \\
\hline Mês 8 & $R \$ 5.288$ & & \\
\hline
\end{tabular}

De posse da Equação 6, apuram-se os FP para a unidade, nos horários HFP e HP, conforme demonstrado na Tabela 6 , onde observa-se que realmente esses valores se encontram abaixo do mínimo determinado. A partir da Equação 7, foram calculados os bancos de capacitor para corrigir as situações de FP médios e também para os FP dos piores casos, conforme resultados da Tabela 7, dados em kVAr.

Tabela 6. Fatores de potência apurados para HFP e HP

\begin{tabular}{|c|c|c|c|c|c|}
\hline Mês & FP HFP & FP HP & Mês & FP HFP & FP HP \\
\hline Aug-15 & 0,83 & 0,83 & Apr-16 & 0,77 & 0,80 \\
\hline Sep-15 & 0,85 & 0,87 & May-16 & 0,76 & 0,80 \\
\hline Oct-15 & 0,85 & 0,86 & Jun-16 & 0,75 & 0,79 \\
\hline Nov-15 & 0,85 & 0,86 & Jul-16 & 0,75 & 0,79 \\
\hline Dec-15 & 0,85 & 0,85 & Aug-16 & 0,73 & 0,78 \\
\hline Jan-16 & 0,82 & 0,85 & Sep-16 & 0,72 & 0,80 \\
\hline Feb-16 & 0,76 & 0,77 & MÉDIA: & 0,79 & 0,82 \\
\hline
\end{tabular}

Tabela 7. Dimensionamento do banco de capacitores HFP e HP

\begin{tabular}{ccc}
\hline & F.P. Médio & Pior F.P. \\
\hline Banco HFP - kVAr: & 38,44 & 51,36 \\
\hline Banco HP - kVAr:: & 4,71 & 6,11 \\
\hline
\end{tabular}

Faz-se aqui uma observação importante. Muitas vezes o banco de capacitores com valor adequado já existe na instalação (caso deste estudo), estando simplesmente desativado. Dessa forma, recomenda-se uma conferência simples na subestação. Isso pode eliminar as penalidades com reativo de forma simples, imediata e sem custo. Em alguns casos, no entanto, é necessário adquirir um banco. Capacitores de algumas dezenas de kVAr, para níveis de distribuição, custam em torno de $R \$ 10.000$ a $R \$ 20.000$. Considerando o benefício médio de $R \$ 3.751$ da Tabela 5, esse tipo de investimento apresenta um payback simples em torno de 3 a 5 meses (desconsiderando o custo do banco de capacitores). Um investimento simples e totalmente recomendado para melhorar a eficiência energética da unidade produtiva. 


\section{CONCLUSÃO}

Este trabalho apresentou uma metodologia para redução dos custos de energia elétrica em unidades produtivas, aplicada a um estudo de caso real de uma unidade de distribuição de aço. A metodologia consiste em avaliar a demanda contratada para redefinir o valor contratado, efetuar uma análise tarifária para determinação da modalidade ideal e dimensionar o banco de capacitores para correção do fator de potência.

No estudo de caso, obteve-se uma redução superior a $15 \%$ do custo total. Readequou-se a demanda contratada para $260 \mathrm{KW}$, representando um benefício anual de $R \$ 25.392$. Determinou-se a manutenção da modalidade Verde, caso não haja aumento do consumo HP superior a 15,8\%. Redimensionou-se o banco de capacitores para a correção do fator de potência, gerando um benefício anual de $R \$$ 45.012. Nesse último caso, desmistifica-se o termo "pagamento de reativo" amplamente utilizado pela indústria, haja vista que paga-se na verdade pela potência ativa que a concessionária deixou de receber em função FP abaixo do permitido pela legislação.

As ações demonstradas representam estratégias simples e eficazes na redução de custos, que podem garantir benefícios imediatos e muitas vezes com pouco ou nenhum investimento. Trabalhos desta natureza normalmente são feitos por empresas de consultoria, porém, conforme aqui tratado, há condições de serem realizados pela própria empresa, de maneira confiável e econômica, contribuindo para o aumento da eficiência energética e a produção sustentável no meio siderúrgico. Esse trabalho pretendo contribuir nesse sentido.

\section{REFERÊNCIAS}

1 Programa Nacional de Conservação de Energia Elétrica - PROCEL. Manual de Tarifação da Energia Elétrica.2001 [acesso em 25 nov. 2016];1:5-11. Disponível em: http://www.sef.sc.gov.br/sites/default/files/manual de tarifacao.pdf

2 Comitê de Distribuição de Energia Elétrica - CODI. Manual de Orientação aos Consumidores. Energia Reativa Excedente. 2004 [acesso em 28 nov. 2016];1:5-11. Disponível em:

http://www.edp.com.br/distribuicao/edp-bandeirante/informacoes/grandesclientes/normas-e-manuais/Documents/Manual\%20de\%20Orientação\%20Energia\%20Reativa\%20Excedente.pdf

3 Agência Nacional de Energia Elétrica- ANEEL. "Resolução Normativa n 414"; 2010.

4 Agência Nacional de Energia Elétrica - ANEEL. "Procedimentos de distribuição de Energia Elétrica no Sistema Elétrico Nacional - PRODIST", 2016.

5 Agência Nacional de Energia Elétrica - ANEEL. "Resolução Normativa no 2.076"; 2016. 